\title{
A case of continuous-type splenogonadal fusion
}

\author{
Jasin Arachchige Saman Bingumal Jayasundara ${ }^{1}$, MD, MRCs, Vithanage Hasanthi Vithana ${ }^{1}$, MD, MRCs, \\ Ananda Kumara Lamahewage ${ }^{1}$, MS, FRCS
}

\begin{abstract}
Splenogonadal fusion is a rare developmental anomaly in which an abnormal connection between the splenic tissue and gonads or mesonephric derivatives is present. Less than 200 cases have been reported since it was first described in 1883. Preoperative misdiagnosis is common and may lead to unnecessary orchidectomy if testicular neoplasm is suspected. To avoid such outcomes, it is important to be aware of the features of splenogonadal fusion. We report the case of a five-month-old male infant with continuous-type, left-sided splenogonadal fusion, which was discovered during groin exploration for a scrotal mass. Although the lesion was first noted during an episode of nonspecific viral fever, such an association is uncommon. Preoperative ultrasonographic evaluation favoured a diagnosis of a large haemangioma. This is the first reported case of splenogonadal fusion from Sri Lanka.
\end{abstract}

Keywords: developmental anomaly, ectopic spleen, orchidectomy, splenogonadal fusion

\section{INTRODUCTION}

Splenogonadal fusion is a rare developmental anomaly in which an abnormal connection between the splenic tissue and gonads or mesonephric derivatives is present. Less than 200 cases have been reported since it was first described in 1883 by Bostroem. ${ }^{(1)}$ Preoperative misdiagnosis had resulted in unnecessary orchidectomy in about a third of reported cases. ${ }^{(2)}$ Thus, to avoid such outcomes, awareness of this rare entity among paediatric and urological surgeons is needed. There are two types of splenogonadal fusion - continuous and discontinuous. Several developmental anomalies have been reported to be associated with continuous-type splenogonadal fusion. We report a case of continuous-type, left-sided splenogonadal fusion in a fivemonth-old male infant, discovered during groin exploration. The initial presentation was that of a scrotal mass noted during an episode of viral fever, which is a rare presentation of splenogonadal fusion.

\section{CASE REPORT}

A five-month-old male infant presented to the paediatric surgery clinic with a left-sided scrotal swelling that had persisted for two months. The lesion was first discovered by the child's mother due to the warmth and redness of the child's scrotum during an episode of nonspecific viral fever at three months of age. As the scrotal warmth and fever resolved with conservative management, the child was not brought to a physician at that time. However, as the swelling persisted, the parents eventually sought medical advice.

The patient was asymptomatic at the time of the review. Physical examination revealed a mass in the left hemiscrotum. The mass was palpable almost up to the superficial inguinal ring, and the testis was not separately palpable. The rest of the external genitalia were normal and systemic examination findings were unremarkable. Sonographic evaluation of the groin and scrotum

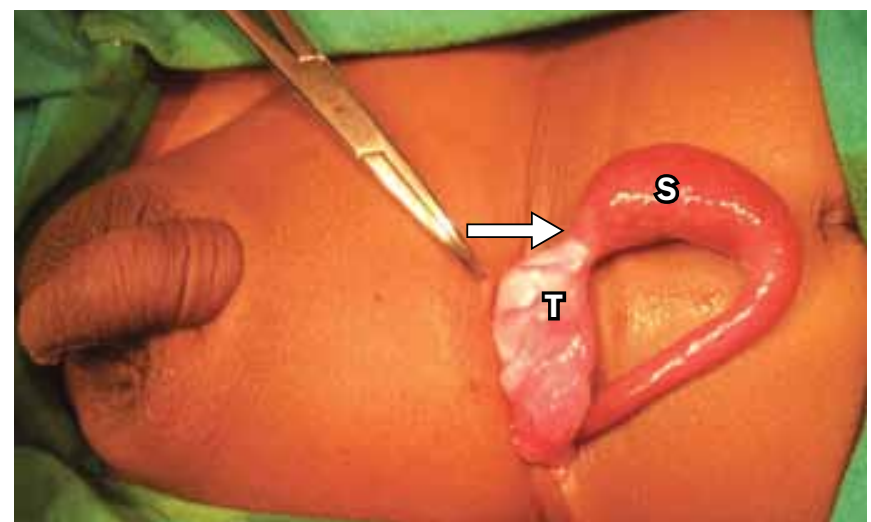

Fig. 1 Perioperative photograph shows the splenic cord (S) joined to the testicle $(T)$ at the fusion point (arrow), confirming the diagnosis of continuous-type splenogonadal fusion.

were suggestive of a large haemangioma. Surgical exploration of the left groin revealed a normal-looking spermatic cord and another smooth, fleshy, brownish, cord-like structure travelling through the processus vaginalis. Following delivery of the testis into the groin incision, a diagnosis of continuous-type splenogonadal fusion was evident (Fig. 1). Distally, the cord-like splenic tissue was attached to the testis, and proximally, it was continuous and connected to the principal spleen.

The splenogonadal fusion was divided using bipolar electrocautery, and the cord of the splenic tissue was divided deep to the deep inguinal ring after ligation. The resected segment was sent for histological assessment. Herniotomy was done and the testicle was repositioned into the scrotum. The patient had an uncomplicated postoperative period. Histological evaluation of the specimen showed normal splenic tissue.

\section{DISCUSSION}

The first description of splenogonadal fusion was made by Bostroem in 1883, and a detailed report by Pommer followed

${ }^{1}$ Department of Paediatric Surgery, Lady Ridgeway Hospital for Children, Colombo, Sri Lanka

Correspondence: Dr JASB Jayasundara, Registrar, Department of Paediatric Surgery, Lady Ridgeway Hospital for Children, Colombo 8, 00800, Sri Lanka bingumal@sItnet.Ik 
in 1889. ${ }^{(1)}$ Less than 200 cases have been reported worldwide since its first description. In 1990, a detailed literature review of 123 cases was conducted by Carragher. ${ }^{(1)}$

In a review of 30 cases, Putschar et al categorised splenogonadal fusion into continuous and discontinuous types, depending on the anatomical continuity between the principal spleen and the gonad. ${ }^{(3)}$ In the continuous type, a cord of splenic tissue, a fibrous cord beaded with splenic nodules or a pure fibrous band anatomically connects the main spleen to the gonad. This cord may traverse the peritoneal cavity to enter the inguinal canal or remain in the paracolic gutter. In the discontinuous type, the ectopic splenic tissue would be adherent to the gonads with no anatomical connections to the principal spleen. Carragher reported that both types occur with equal frequency. ${ }^{(1)}$ However, Le Roux et $\mathrm{al}^{(4)}$ suggested that discontinuous-type splenogonadal fusion is a rare variant of accessory spleen.

Splenogonadal fusion has commonly been an incidental finding during groin exploration for undescended testis or hernia. About a sixth of all cases of splenogonadal fusion were found during autopsies. ${ }^{(2)}$ This malformation has predominantly been reported in males, with a male to female ratio of $16: 1$, according to Carraghers' review. ${ }^{(1)}$ Nonrequirement for, and inaccessibility to, clinical examination of the female gonads (compared to undescended testis), however, may have masked the true prevalence of asymptomatic splenogonadal fusion in females. Although the left testis is predominantly involved, cases of right splenogonadal fusion have also been reported. ${ }^{(5,6)}$

Splenogonadal fusion may present as painless testicular swelling or acute scrotal swelling. Some of the symptomatic cases that present as painless testicular swelling may be misdiagnosed as neoplasms. In a cumulative case review, Karaman et al found that misdiagnosis of testicular swelling as testicular neoplasm resulted in orchidectomy in more than $30 \%$ of cases reviewed.(2) To date, only a few cases of splenogonadal fusion presenting as acute scrotal swelling have been reported, one of which was a study by Settle, ${ }^{(7)}$ which reported two patients who presented with acute scrotal swelling following malaria. Infectious mononucleosis, torsion of the splenic cord and traumatic rupture of the ectopic splenic tissue have also been reported to cause acute scrotum in splenogonadal fusion. . $^{(2,8,9)}$ Hines et al found that bowel obstruction can occur due to the intraperitoneal segment of a continuous splenogonadal fusion. ${ }^{(10)}$ In the case of our five-month old patient, although the correct diagnosis was confirmed via surgical exploration at a later stage, the lesion was first identified during an episode of nonspecific viral fever, which is not common.

Several cases of testicular malignancies in undescended testis associated with splenogonadal fusion have been reported. ${ }^{(11,12)}$ Although preoperative diagnosis of splenogonadal fusion using imaging modalities is rare, ultrasonography and splenic scintigraphy have been successfully used. ${ }^{(13)}$ In our case, sonography suggested a haemangioma, probably due to the high vascularity of the splenic tissue. Splenogonadal fusion has been associated with many anomalies, including cryptorchidism, which has been found in a third of cases of splenogonadal fusion. ${ }^{(14)}$ Other associated anomalies include peromelia, micrognathia, hypoglossia, craniosynostosis, spina bifida, palatine and cardiac defects, as well as limb, anorectal and rare syndromic anomalies. ${ }^{(1,2,5)}$ While these anomalies are more common in patients with continuous-type splenogonadal fusion, it was fortuitous that our patient did not have any associated anomalies.

The aetiology of splenogonadal fusion is still not clearly understood. It is embryologically explained as the fusion between the primitive splenic anlage and the developing mesonephric-gonadal structures, in the fifth to eighth weeks of intrauterine life. ${ }^{(4)}$ Although several hypotheses have been postulated regarding the mechanism by which splenogonadal fusion arises, no consensus on the exact mechanism has been reached. ${ }^{(1,14)}$

In conclusion, splenogonadal fusion is a rare congenital anomaly with equivocal clinical presentations that may mislead clinicians. As basic imaging modalities may not aid in the accurate diagnosis of this condition, it is critical that both paediatric and urological surgeons are aware of this disease entity so that an early and accurate diagnosis may be reached, thus avoiding unnecessary orchidectomies.

\section{REFERENCES}

1. Carragher AM. One hundred years of splenogonadal fusion. Urology 1990; 35:471-5.

2. Karaman MI, Gonzales ET Jr. Splenogonadal fusion: report of 2 cases and review of the literature. J Urol 1996; 155:309-11.

3. Putschar WG, Manion WC. Splenicgonadal fusion. Am J Pathol 1956; 32:15-33.

4. Le Roux PJ, Heddle RM. Splenogonadal fusion: is the accepted classification system accurate? BJU Int 2000; 85:114-5.

5. Khairat $A B$, Ismail AM. Splenogonadal fusion: case presentation and literature review. J Pediatr Surg 2005; 40:1357-60.

6. Brash J, Roscher AA. Unusual presentation on the right side of ectopic testicular spleen. Int Surg 1987; 72:233-4.

7. Settle EV. The surgical importance of accessory spleens with a report of 2 cases. Am J Surg 1940; 50:22-6.

8. Andrews RW, Copeland DD, Fried FA. Splenogonadal fusion. J Urol 1985; 133:1052-3.

9. Halvorsen JF, Stray O. Splenogonadal fusion. Acta Paediatr Scand 1978; 67:379-81.

10. Hines JR, Eggum PR. Splenic-gonadal fusion causing bowel obstruction. Arch Surg 1961; 83:887-9.

11. Falkowski WS, Carter MF. Splenogonadal fusion associated with an anaplastic seminoma. J Urol 1980; 124:562-4.

12. Imperial SL, Sidhu JS. Nonseminomatous germ cell tumor arising in splenogonadal fusion. Arch Pathol Lab Med 2002; 126:1222-5.

13. Steinmetz AP, Rappaport A, Nikolov G, et al. Splenogonadal fusion diagnosed by spleen scintigraphy. J Nucl Med 1997; 38:1153-5.

14. Cortes D, Thorup JM, Visfeldt J. The pathogenesis of cryptorchidism and splenogonadal fusion: a new hypothesis. Br J Urol 1996; 77:285-90. 